\title{
Resultados adversos en la procreación en mujeres trabajadoras en la floricultura colombiana: un resumen de la evidencia mediante metanálisis
}

\author{
Álvaro Javier Idrovo', Luz Helena Sanín ${ }^{1,2}$ \\ ${ }^{1}$ Instituto Nacional de Salud Pública, Cuernavaca, Morelos, México. \\ 2 FEN/Universidad Autónoma de Chihuahua, Chihuahua, México.
}

Introducción. Los trabajadores en la floricultura están expuestos a plaguicidas y otros factores de riesgo. Se sospecha que el trabajo en estos ambientes ocupacionales está asociado con resultados adversos en la procreación. Sin embargo, los estudios epidemiológicos en Colombia, el país de América Latina con más vieja tradición con estos cultivos, no son consistentes ni concluyentes.

Objetivo. Cuantificar la relación entre el trabajo de las mujeres en la floricultura colombiana con la aparición de abortos espontáneos, partos prematuros y malformaciones congénitas, usando los resultados de dos estudios previos.

Materiales y métodos. Mediante técnicas de metanálisis se resumieron los resultados de dos estudios con poblaciones trabajadoras en la floricultura colombiana. Se llevó a cabo una evaluación crítica de cada uno de los estudios para identificar los potenciales sesgos y limitaciones metodológicas.

Resultados. El estimador agregado para abortos espontáneos fue de 2,24 (IC95\%: 1,872,68), para prematuros fue de 1,49 (IC95\%: 0,91-2,43) y para malformaciones congénitas de 1,31 (IC95\%: 1,05-1,64).

Conclusiones. Los análisis sugieren que el trabajo en la floricultura se asocia con una mayor aparición de abortos espontáneos y malformaciones congénitas. Sin embargo, no hay indicios de que estos efectos estén asociados con la exposición a plaguicidas. Otros factores de riesgo que requieren posterior estudio son la actividad física y el calor dentro de los invernaderos, ambos presentes en las empresas floricultoras. La evidencia de los países que han entrado recientemente a la industria, ayudará a dilucidar esta relación.

Palabras clave: plaguicidas/efectos adversos, agricultura, aborto espontáneo, prematuro, metanálisis, Colombia.

Adverse reproductive outcomes among women working in Colombian floriculture: a summary of the evidence through metaanalysis

Introduction. Workers in floriculture are exposed to pesticides and other risk factors. Work in these occupational environments is suspected to be associated with adverse reproductive outcomes. However, epidemiological studies in Colombia, a country that has a long tradition with this occupation, have not been consistent or conclusive.

Objective. To quantify the effect of women's work in Colombian floriculture in the occurrence of spontaneous abortion, prematurity, and birth defects, using the results of two previous studies. Material and methods. The results of two previous studies among Colombian floriculture worker populations were summarized with metaanalysis techniques. A critical evaluation of each of the studies identified potential biases and methodological limitations.

Results. The pooled estimator of spontaneous abortion was 2.24 (95Cl: $1.87-2.68$ ); that of premature infant birth was $1.49(95 \% \mathrm{Cl}: 0.91-2.43)$, and of birth defects 1.31 (95\% Cl: $1.95-$ 1.64).

Conclusions. The analyses suggested that work in floriculture is associated with a higher occurrence of spontaneous abortion and birth defects. However, these effects are not necessarily associated with pesticide exposure. Other risk factors requiring further study are levels of 
physical activity and high temperature in greenhouses. Data from countries which have initiated occupations in floriculture more recently will add valuable evidence for this controversial association.

Key words: pesticides/adverse effects; agriculture; abortion, spontaneous; infant/premature; metaanalysis, Colombia.

Desde hace más de 20 años se postula que el trabajo en la floricultura colombiana ocasiona efectos adversos en la procreación, especialmente debido a la exposición a las mezclas de plaguicidas. Esta preocupación propició la realización de estudios de higiene ocupacional (1-3), y varios estudios epidemiológicos en esta población para explorar específicamente esta problemática (4-6), así como algunos posibles efectos cancerígenos $(7,8)$. Los resultados obtenidos hasta ahora no son concluyentes y, siguen los patrones observados en estudios similares realizados con poblaciones agrícolas expuestas a plaguicidas de otras regiones del mundo $(9,10)$. Es decir que existen datos que apoyan una relación causal entre la exposición a plaguicidas, $u$ otros factores de riesgo presentes en estos ambientes ocupacionales, y la presencia de resultados adversos en la procreación, así como hay estudios que muestran resultados contrarios.

El hecho de contar con dos estudios observacionales realizados en empresas floricultoras colombianas que han abordado el tema, y presentan similar metodología, brindó la oportunidad de resumir la evidencia disponible, buscando así obtener un cuadro más real de la situación. Esta población resulta de especial interés ya que por las condiciones propias del trabajo en invernaderos podrían tener una mayor exposición a los plaguicidas, por lo que se esperaría una mayor frecuencia de efectos adversos en comparación con otras poblaciones expuestas en actividades agrícolas. El objetivo del presente estudio fue resumir cuantitativamente y analizar cualitativamente la información disponible en torno a tres de los efectos reproductivos más controversiales: los

\footnotetext{
Correspondencia:

Alvaro Javier Idrovo, Instituto Nacional de Salud Pública, Avenida Universidad 803, Colonia Santa María, Ahuacatitlán, CP 62100, Cuernavaca, Morelos, México.

Tel. 52 (777) 329 300, ext. 5353

ajidrovo@correo.insp.mx

Recibido: 08/11/06; aceptado: 01/08/07
}

abortos espontáneos, los partos prematuros y las malformaciones congénitas, utilizando los datos recolectados en los dos estudios previos con esta población.

\section{Material y métodos}

Se realizó un análisis agregado de los dos estudios que han explorado el potencial impacto del trabajo en la floricultura en los resultados en la procreación. Se utilizaron parte de los datos descritos en el estudio de Restrepo et al. (4), junto con los aportados por el estudio de Idrovo y colaboradores (enviado para publicación) cuya metodología, parcialmente, se encuentra en otra publicación (6).

El primer estudio, en 1981, tuvo un diseño transversal y recolectó información mediante un cuestionario precodificado a 3.489 trabajadoras de empresas floricultoras de la sabana de Bogotá, obteniendo información de 10.481 embarazos; de las trabajadoras que fueron reportadas activas en ese momento por las empresas, sólo se excluyeron aquéllas que reportaron estar enfermas, en vacaciones o se habían retirado del trabajo en el momento en que se llevó a cabo la entrevista personal (4).

El segundo estudio también fue transversal y tuvo como resultado primario el tiempo para quedar en embarazo, el cual es un reconocido indicador de baja fecundidad (11). En este caso, la información fue recolectada en 2001 a las mujeres trabajadoras de 47 empresas floricultoras de la sabana de Bogotá. Sólo se incluyeron los primeros embarazos de las mujeres que reportaron haber tenido un embarazo, sin importar el resultado en los 10 años previos a la entrevista. Se entrevistaron $2.283 \mathrm{mu}$ jeres, pero sólo se incluyeron 1.948 en los análisis aquí descritos ya que se excluyeron las que estaban usando un método anticonceptivo hormonal en el año previo al embarazo, las que tuvieron datos faltantes en la historia laboral y de potenciales determinantes claves relacionados con el tiempo para quedar en embarazo (11). Dado 
que este estudio sólo recolectó información de las mujeres trabajadoras, los análisis agregados, aquí presentados, únicamente incluyen la información sobre la exposición de las mujeres, más no de los hombres. Una descripción más completa de los estudios se puede encontrar en otras publicaciones $(4,6)$.

\section{Objetos de estudio}

Los dos estudios mencionados tuvieron exactas definiciones de aborto espontáneo, prematuridad y malformación congénita. Se consideró que hubo aborto espontáneo si la mujer reportaba una pérdida fetal menor de 20 semanas de gestación, prematuro si se reportaba un nacido vivo entre la semana 21 y la 36 de gestación, y malformado si se reportaba un defecto estructural o funcional detectado por los por los padres o por un médico en el momento del nacimiento o después.

Estas formas de medir los abortos espontáneos y la prematuridad han sido objeto de estudio en otras regiones, y se sabe que presentan especificidades superiores al $99 \%$, aunque su sensibilidad puede tener valores entre $63 \%$ y $74 \%$, aproximadamente (12-14), de acuerdo con las poblaciones estudiadas. El caso de las malformaciones congénitas fue abordado explícitamente en estas poblaciones, y se sabe que su sensibilidad es de $69,4 \%$ y su especificidad de $75,1 \%$ (15). Pese a esto, se considera que la información brindada por las mujeres tiene unos niveles de confiabilidad y precisión aceptables (14), teniendo en cuenta los bajos costos requeridos para su recolección, cuando se hacen estudios epidemiológicos con poblaciones numerosas. Como es bien conocido, el efecto de estos errores de medición no diferenciales corresponde casi siempre a la atenuación de las asociaciones (16).

\section{Evaluación de la exposición}

La medición de la exposición en cuanto a plaguicidas específicamente presenta problemas en los dos grupos estudiados ya que no hay un biomarcador de exposición subcrónica y deben limitarse al cuestionario; sin embargo, la exposición ocupacional como un todo que incluye diferentes riesgos y exigencias, es evaluada de manera similar en ambos grupos, considerando tanto antigüedad, como tipo de actividad y, por ello, es mas cercana esta última a una verdadera medición de la exposición.

\section{Evaluación crítica de los estudios}

Un paso indispensable cuando se realizan metanálisis es la evaluación crítica de los estudios; si bien existe un desarrollo importante al respecto para el caso de los ensayos clínicos, en el caso de los estudios observacionales no hay criterios universalmente aceptados para evaluar su calidad. Debido a ello, y con el objetivo de hacer una revisión crítica y estandarizada de los estudios, en este análisis se siguieron los criterios para la evaluación de estudios observacionales longitudinales de Tooth et al. (17), similar a los utilizados en la revisión sistemática de Sanborns et al. (10); de los 33 criterios originales no se analizaron cinco, debido a que son explícitos de estudios de seguimiento. Estos criterios se encuentran listados en el cuadro 1.

\section{Métodos estadísticos}

Para resumir los estimadores de asociación obtenidos en los estudios, primero se evaluó su heterogeneidad con la prueba de DerSimonian y Laird (18). De esta manera, se pudo observar que, en los casos del aborto espontáneo y las malformaciones congénitas, los resultados eran homogéneos $(p>0,68)$, mientras que para los partos prematuros no $(p=0,014)$. Por tal razón, para obtener los estimadores se usaron modelos de efectos fijos de Mantel-Haenszel para los abortos espontáneos y las malformaciones congénitas, y uno de efectos aleatorios de DerSimonian y Laird para los partos prematuros (18). Debido a que sólo se tienen dos estudios no fue posible ajustar los estimadores obtenidos por las diferencias metodológicas de los estudios mediante técnicas de metaregresión, evaluar el sesgo de publicación o realizar análisis formales de sensibilidad. En estos análisis se utilizó el programa estadístico Stata 9 (Stata Corporation, College Station), con macros desarrollados por Bradburn, Deeks y Altman.

\section{Resultados}

Una evaluación crítica de los estudios se encuentra en el cuadro 1. Allí se puede apreciar 
Cuadro 1. Resultados de la revisión crítica de los estudios incluidos en los metanálisis, basados en los criterios de Tooth et al.

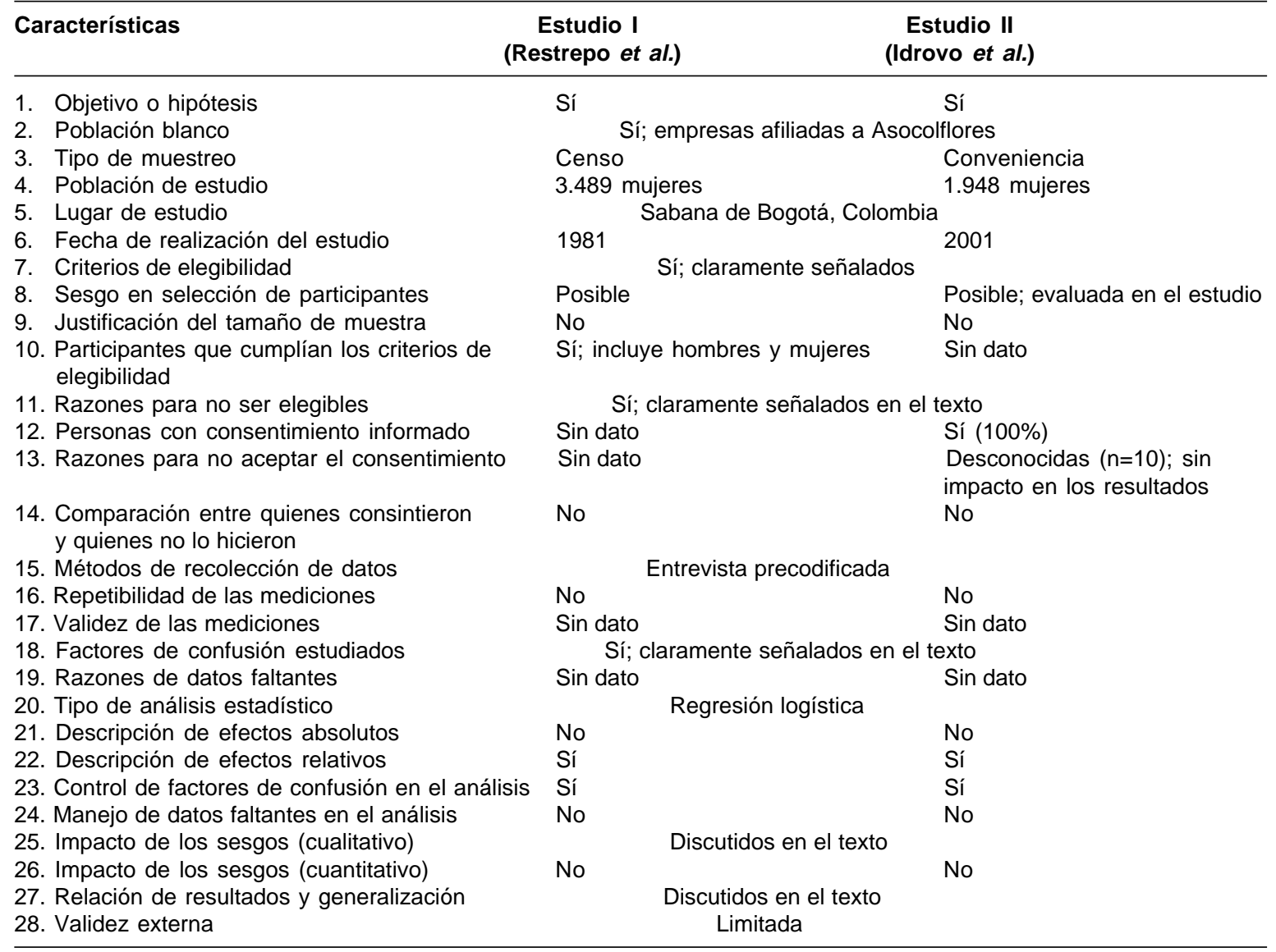

que los estudios pese a tener diferencias en el diseño, presentan muchas características similares que permiten agregar los resultados obtenidos independientemente; en especial, presentan algunos potenciales problemas metodológicos asociados, en gran medida, a los estudios transversales $(19,20)$ y a la temática de la procreación (21). En relación con los estimadores agregados, en el caso de los abortos espontáneos se obtuvo una razón de momios de 2,24 (IC95\%: 1,87-2,68), lo cual indica que una mujer al estar trabajando en la floricultura incrementa un poco más de dos veces la posibilidad de presencia de abortos espontáneos, cuando se compara con las mujeres que no están en la floricultura (figura 1). La razón de momios resumen para los prematuros fue de 1,49 (IC95\%: 0,91$2,43)$, que sugiere que no existen diferencias estadísticamente significativas $(p<0,05)$ en la frecuencia de partos prematuros cuando se comparan las mujeres que trabajan en la floricultura con las que no lo hacen (figura 2). En el caso de las malformaciones congénitas se obtuvo una razón de momios de 1,31 (IC95\%: $1,05-1,64)$, la cual apoya la idea que las mujeres que trabajan en la floricultura tienen una posibilidad mayor de tener niños con malformaciones congénitas, que las que no trabajan allí (figura 3).

\section{Discusión}

Los resultados cuantitativos sugieren que sí existe un efecto de trabajar en la floricultura sobre los resultados de la procreación, especialmente en lo relacionado con los abortos espontáneos y las malformaciones congénitas. Estos hallazgos, sin embargo, deben interpretarse con cautela dadas 


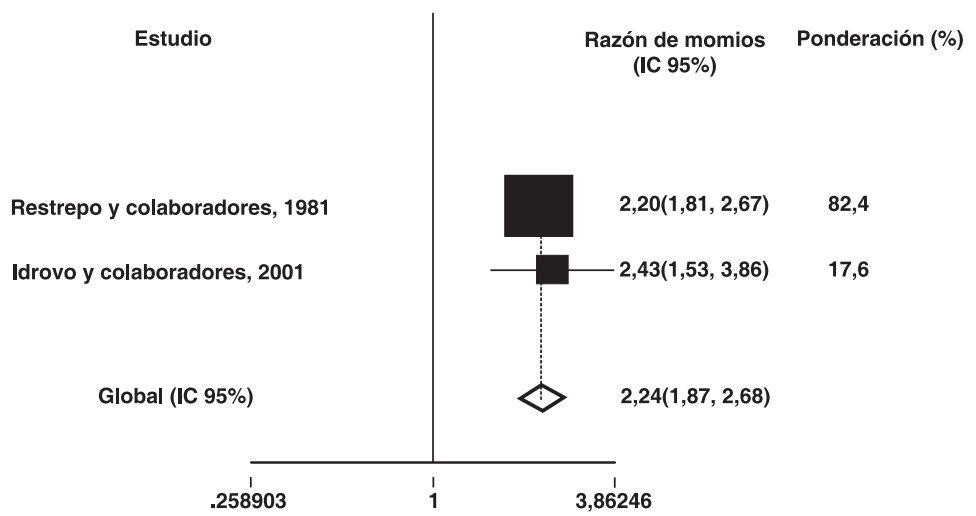

Figura 1. Diagrama de bosque para resumir los estudios epidemiológicos que estudian la prevalencia de abortos espontáneos en la floricultura colombiana.

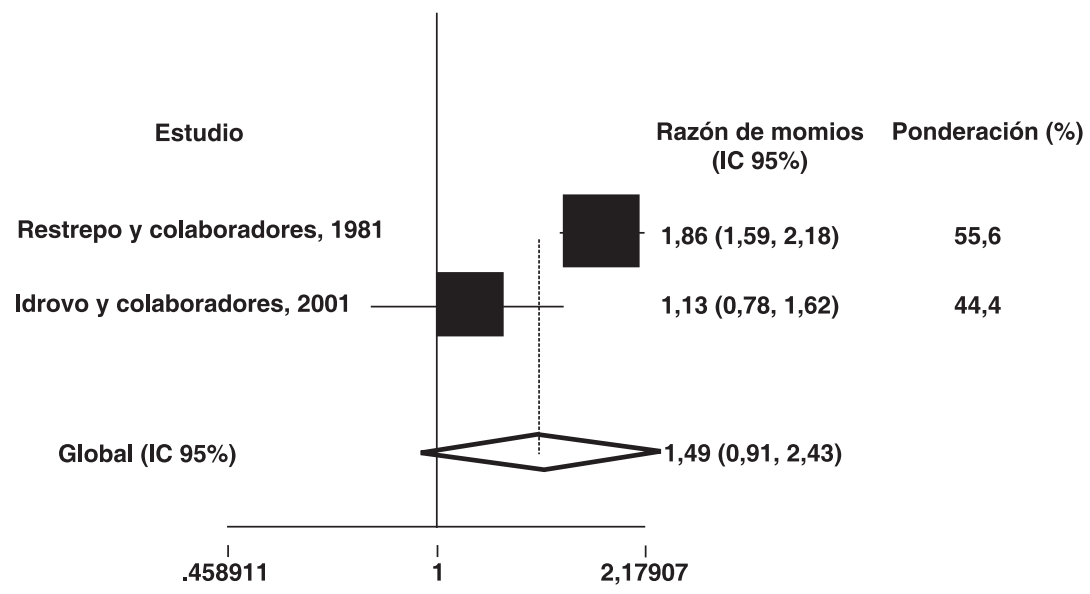

Figura 2. Diagrama de bosque para resumir los estudios epidemiológicos que estudian la prevalencia de partos prematuros en la floricultura colombiana.

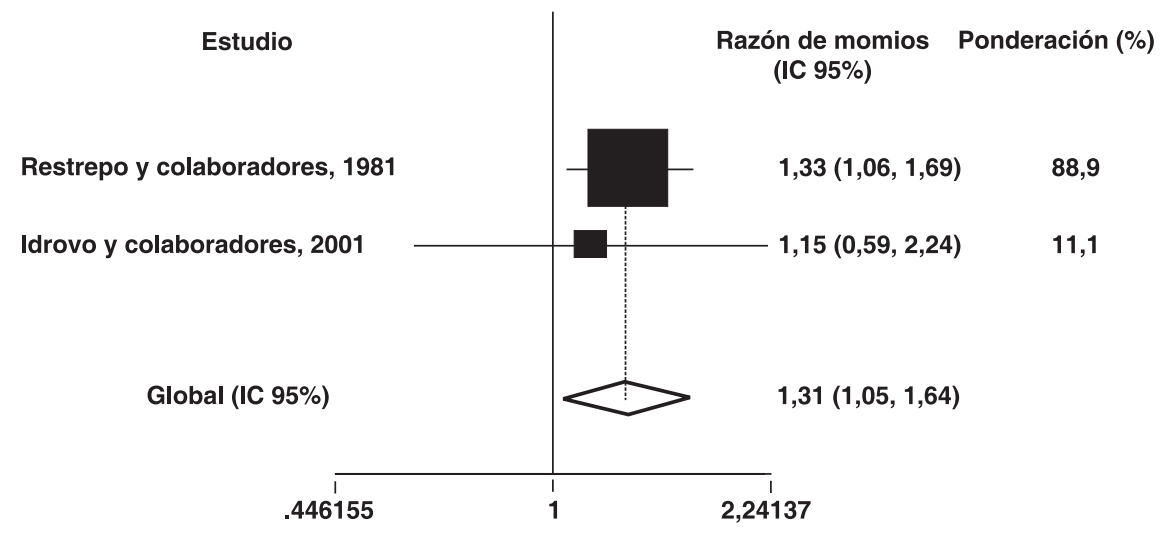

Figura 3. Diagrama de bosque para resumir los estudios epidemiológicos que estudian la prevalencia de malformaciones congénitas en la floricultura colombiana. 
las limitaciones inherentes de los estudios observacionales que brindaron los datos aquí analizados. El análisis cualitativo permite señalar que las diferencias metodológicas en los diseños, no son mayores como para no poder analizar los datos; quizá la diferencia más importante radica en que en un estudio se evalúa más de un embarazo por mujer, mientras que en el otro, sólo se incluyeron los primeros embarazos para evitar la confusión potencial relacionada con la historia reproductiva previa. Esta ha sido señalada como una de las principales limitaciones en los estudios de epidemiología de la reproducción $(11,21)$. De otro lado, debe recordarse que las técnicas de metanálisis son especialmente útiles cuando se tiene un número importante de estudios; sin embargo, éste no es el caso ya que sólo existen dos estudios comparables disponibles, pero dada la ausencia de otros casos, se consideró que los datos se podían combinar.

Los estimadores agregados obtenidos para los abortos espontáneos, la prematuridad y las malformaciones congénitas, junto a los otros efectos reproductivos adversos estudiados en sólo uno de los estudios, permiten tener una panorámica más sólida de esta problemática en la floricultura colombiana. En la figura 4 se encuentra un iceberg que sirve para representar el número proporcional de efectos adversos, junto con los estimadores obtenidos en los estudios epidemiológicos. Como se puede apreciar, los eventos más frecuentes son los abortos espontáneos no reconocidos por la mujer y los reconocidos clínicamente (por la mujer o por un profesional de la salud). Éstos se pueden estudiar mediante el método de tiempo para quedar en embarazo (11) o el reporte de su suceso; en este último caso, sólo se obtiene información de los embarazos y abortos clínicamente reconocidos, lo que puede ocasionar una subestimación del efecto. Con el método de tiempo para quedar en embarazo se tiene una mejor aproximación a los abortos no reconocidos. Los resultados aquí descritos, junto con los estimadores ajustados presentados en los estudios originales, son consistentes en mostrar que existe un mayor riesgo de presencia de abortos, reconocidos y no reconocidos clínicamente, entre las trabajadoras de empresas floricultoras.

En relación con los mortinatos y los prematuros, los datos no muestran consistencia y los estimadores tienden a estar muy cerca del valor de nulidad. Por esta razón, la conclusión es que estos efectos no se encuentran asociados con el trabajo en empresas floricultoras. Esto puede ser debido a que después de ser reconocido un embarazo, por políticas de salud ocupacional en las empresas, a las mujeres se les cambia su actividad, buscando actividades menos extenuantes y con menor riesgo de exposición a los plaguicidas.

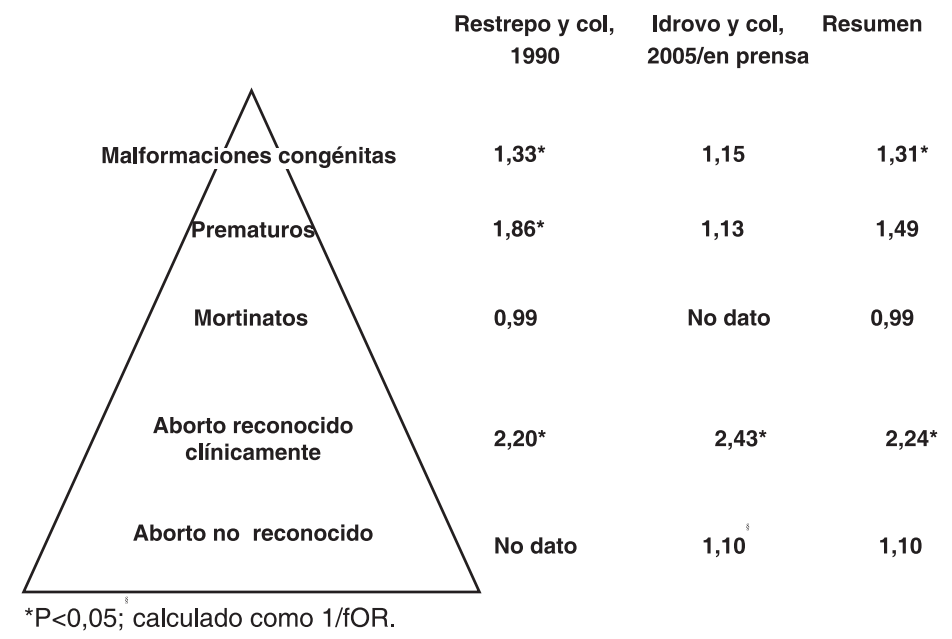

Figura 4. Iceberg de los principales efectos adversos en la procreación y su relación con el trabajo en la floricultura colombiana. 
Finalmente, con las malformaciones congénitas los resultados agregados sugieren un efecto negativo del trabajo en la floricultura. Sin embargo, debe tenerse en cuenta que la forma de medición de estos hechos crea un efecto de mala clasificación, que puede ser corregido cuando se hacen análisis específicos para cada tipo de malformación (5); sin embargo, esto no fue posible de realizar en el estudio de Idrovo et al. debido a la baja frecuencia de casos.

Aun con dicho problema y considerando que la etiopatogenia de las malformaciones congénitas se asocia en gran medida con exposiciones en las primeras semanas del embarazo, de manera similar a los abortos espontáneos reconocidos y no reconocidos clínicamente, los hallazgos muestran consistencia en relación a una ventana de exposición: la que ocurre entre la concepción y el reconocimiento del embarazo; este análisis se hace a partir de la interpretación de la prolongación del tiempo para embarazo como una medida indirecta de las pérdidas tempranas que pueden ocurrir por el motivo descrito. Esto debe interpretarse como que las mujeres que requieren mayor atención son las que están en riesgo de lograr una concepción. Este tipo de conclusiones ya han sido obtenidas en estudios recientes que abordan el tema de las anencefalias entre poblaciones expuestas a plaguicidas en México (22). El tiempo para quedar en embarazo cobra especial importancia en este grupo como evento centinela que puede alertar sobre posibles riesgos, ya que uno de los primeros efectos que puede detectar es el de pérdidas tempranas, no reconocidas por la madre. Además es un indicador fácil de obtener, validado, no invasivo y de bajo costo.

Sin embargo, estos resultados no indican que los efectos son debidos a los plaguicidas; realmente, en las condiciones laborales de las empresas floricultoras existen conjuntamente varios riesgos y exigencias laborales, difíciles de desligar mediante los métodos epidemiológicos convencionales (23). Recientemente, en un estudio realizado con trabajadores en invernaderos, en Polonia, se observó que las mujeres con carga física superior a $1.000 \mathrm{kcal}$ por turno tienen hijos con menor peso al nacer, en comparación con quienes tienen cargas laborales con menor carga física, y que los plaguicidas no muestran este mismo efecto (24). Nuestra sugerencia, basada en el principio de precaución, es que los programas de salud ocupacional de las empresas floricultoras deberían incluir políticas que permitan identificar a las mujeres que buscan una concepción y las embarazadas, con el fin de retirarlas de los invernaderos, donde se juntan varios factores de riesgo que pueden actuar sinérgicamente incrementando el riesgo de presencia de abortos y malformaciones congénitas.

En conclusión, los datos epidemiológicos sugieren un posible efecto nocivo del trabajo en la floricultura, pero no permite sostener que sea debido a los plaguicidas $u$ otras condiciones propias de la población que allí labora. Futuros estudios podrán complementar esta información cuantitativa con la obtenida en los estudios cualitativos (25) y otras aproximaciones epistemológicas (26), para así obtener una visión más real de esta problemática sanitaria. Los estudios que comienzan a realizarse en países, como Ecuador, México y Perú $(27,28)$, con una industria de las flores más reciente y con diferentes características, pero que ya tienen en su haber una experiencia surgida de estudios en otras regiones, como Colombia, aportarán elementos que ayuden a dilucidar esta asociación.

\section{Conflicto de intereses}

Los autores participaron en uno de los estudios incluidos en el metanálisis.

\section{Financiación}

Este estudio fue patrocinado por la Universidad Autónoma de Chihuahua, instituciones en las cuales laboraban los autores durante la realización del estudio, junto a Colciencias (contrato 1122000) y la División de Investigaciones (DIB) de la Universidad Nacional de Colombia, sede Bogotá.

\section{Referencias}

1. Morgade C, Davies J, Barquet A, Danauskas J, Pfaffenberger C. Captan fungicide and other pesticides exposure of carnation floriculturists in Bogotá, Colombia (Technical Report). Miami: University of Miami School of Medicine; 1983.

2. Barreto-Charry AV, Castiblanco-Rodríguez AL, Ospina de Nigrinis S, Idrovo AJ. Implementación y 
validación de una metodología analítica para la determinación de etilentiourea en orina y parches de extracción. Rev Colomb Cienc Quim Farm. 2003; 32:51-7.

3. Varona ME, Tolosa JE, Cárdenas O, Torres $\mathrm{CH}$, Pardo D, Carrasquilla G, et al. Descripción del uso y manejo de plaguicidas en las empresas de flores afiliadas a Asocolflores. Biomédica. 2005;25:377-89.

4. Restrepo M, Muñoz N, Day NE, Parra JE, de Romero L, Nguyen-Dinh X. Prevalence of adverse reproductive outcomes in a population occupationally exposed to pesticides in Colombia. Scand J Work Environ Health. 1990;16:232-8.

5. Restrepo M, Muñoz N, Day NE, Parra JE, Hernández CA, Blettner $\mathbf{M}$, et al. Birth defects among children born to a population occupationally exposed to pesticides in Colombia. Scand J Work Environ Health. 1990;16:239-46.

6. Idrovo AJ, Sanín LH, Cole D, Chavarro J, Cáceres $\mathbf{H}$, Narváez $\mathbf{J}$, et al. Time to first pregnancy among women working in agricultural production. Int Arch Occup Environ Health. 2005;78:493-500.

7. Varona M, De la Hoz F, Peña G, Cárdenas 0 . Seguimiento de una cohorte de mujeres trabajadoras en cultivos de flores de la Sabana de Bogotá. Biomédica. 1998;18:30-6.

8. Varona M, Cárdenas $\mathbf{O}$, Crane $\mathbf{C}$, Rocha S, Cuervo G, Vargas J. Prevalencia de alteraciones citogenéticas en trabajadores expuestos a plaguicidas en empresas de flores de Bogotá, Colombia. Biomédica. 2003;23:141 52.

9. Hanke W, Jurewicz J. The risk of adverse reproductive and developmental disorders due to occupational pesticide exposure: an overview of current epidemiological evidence. Int J Occup Environ Health. 2004;17:223-43.

10. Sanborns M, Cole D, Kerr K, Vakil C, Sanín LH, Bassil K. Pesticide literature review. Toronto: The Ontario College of Family Physicians; 2004.

11. Idrovo AJ, Sanín LH, Cole DC. Tiempo para quedar en embarazo: consideraciones generales $y$ metodológicas. Biomédica. 2005;25:398-411.

12. Kristensen $\mathbf{P}$, Irgens LM. Maternal reproductive history: a registry based comparison of previous pregnancy data derived from maternal recall and data obtained during the actual pregnancy. Acta Obstet Gynecol Scand. 2000;79:471-7.

13. Buka SL, Goldstein JM, Spartos E, Tsuang MT. The retrospective measurement of prenatal and perinatal events: accuracy of maternal recall. Schizophr Res. 2004;71:417-26.
14. Tomeo CA, Rich-Edwards JW, Michels KB, Berkey CS, Hunter DJ, Frazier AL, et al. Reproducibility and validity of maternal recall of pregnancy-related events. Epidemiology. 1999;10:774-7.

15. Sanín-Aguirre LH, Restrepo M. Defectos al nacer y pesticidas en Colombia. En: Romieu I, Borja V, Hernández M, editores. Metodología epidemiológica aplicada a estudios de salud ambiental. Cuernavaca: Instituto Nacional de Salud Pública; 2000.p.59-73.

16. Armstrong BG. Effect of measurement error on epidemiological studies of environmental and occupational exposures. Occup Environ Med. 1998;55:651-6.

17. Tooth L, Ware R, Bain C, Purdie DM, Dobson A. Quality of reporting of observational longitudinal research. Am J Epidemiol. 2005;161:280-8.

18. DerSimonian R, Laird N. Meta-analysis in clinical trials. Control Clin Trials. 1986;7:177-88.

19. Hernández B, Velasco-Mondragón HE. Encuestas transversales. Salud Pública Mex. 2000;42:447-55.

20. Mann CJ. Observational research methods. Research design II: cohort, cross sectional, and case-control studies. Emerg Med J. 2003;20:54-60.

21. Weinberg CR, Wilcox AJ. Reproductive epidemiology. In: Rothman KJ, Greenland S, editors. Modern epidemiology. 2nd ed. Philadelphia: Lippincott-Raven Publishers; 1998.p.585-608.

22. Lacasaña $M$, Vázquez-Grameix $H$, Borja-Aburto VH, Blanco-Muñoz J, Romieu I, Aguilar-Garduño C, et al. Maternal and paternal occupational exposure to agricultural work and the risk of anencephaly. Occup Environ Med. 2006;63:649-56.

23. Samet JM. What can we expect from epidemiologic studies of chemical mixtures? Toxicology. 1995;105:307-14.

24. Jurewicz J, Hanke W, Makowiec-Dabrowska T, Sobala W. Exposure to pesticides and heavy work in greenhouses during pregnancy: Does it affect birth weight? Int Arch Occup Environ Health. 2005;78:41826 .

25. Walsh D, Downe S. Meta-synthesis method for qualitative research: A literature review. J Adv Nurs. 2005;50:204-11.

26. Idrovo AJ. Hacia una salud pública pluralista: el caso de los plaguicidas y la salud humana. Rev Salud Pública (Bogotá). 2005;7:349-59.

27. De León J. Situación de las floricultoras con exposición crónica a mezclas de plaguicidas en Morelos, México. Boletín Rapam. 1997;20:10-1.

28. Harari R. Seguridad, salud y ambiente en la floricultura. Quito: Corporación IFA; 2004. 